Case Report

\title{
Nanoparticle Albumin-Bound-Paclitaxel in the Treatment of Metastatic Urethral Adenocarcinoma: The Significance of Molecular Profiling and Targeted Therapy
}

\author{
Yasmin M. Abaza ${ }^{1}$ and Carlos Alemany ${ }^{2}$ \\ ${ }^{1}$ Department of Internal Medicine, Florida Hospital, Orlando, FL 32804, USA \\ ${ }^{2}$ Cancer Institute of Florida, Florida Hospital, 2501 North Orange Avenue, Suite 689, Orlando, FL 32804, USA \\ Correspondence should be addressed to Carlos Alemany; carlos.alemany.md@flhosp.org
}

Received 24 June 2014; Accepted 6 August 2014; Published 18 August 2014

Academic Editor: Fumitaka Koga

Copyright (c) 2014 Y. M. Abaza and C. Alemany. This is an open access article distributed under the Creative Commons Attribution License, which permits unrestricted use, distribution, and reproduction in any medium, provided the original work is properly cited.

Primary urethral cancer is rare and accounts for only $0.003 \%$ of all malignancies arising from the female genitourinary tract. Due to the rarity of this disease, no consensus exists regarding the optimal therapeutic approach. Nanoparticle albumin-boundpaclitaxel has been shown to be effective in the treatment of a number of malignancies including metastatic breast, pancreatic, and bladder cancer. We present a 67-year-old woman with advanced metastatic urethral adenocarcinoma resistant to two lines of chemotherapy (ifosfamide/paclitaxel/cisplatin and irinotecan/5-fluorouracil/leucovorin) that showed a dramatic response to nanoparticle albumin-bound-paclitaxel. This is the first case report to document the use and efficacy of nanoparticle albuminbound-paclitaxel in the treatment of unresectable metastatic urethral cancer.

\section{Introduction}

Primary urethral cancer is rare and accounts for approximately $0.02 \%$ of all female cancers [1]. The most common histological type of urethral cancer is transitional cell carcinoma (TCC) followed by squamous cell carcinoma (SCC) and adenocarcinoma [2]. About 98\% of women are symptomatic at presentation complaining of obstructive or irritative voiding symptoms, hematuria, dyspareunia, and a palpable urethral mass [3]. Since the clinical presentation is nonspecific, diagnosis is often delayed until advanced stages of the disease. Due to the rarity of the disease, no consensus exists regarding the optimal therapeutic approach. Nanoparticle albumin-bound (nab) paclitaxel has been shown to be effective in the treatment of metastatic breast, pancreatic, and bladder cancer [4-6]. We report a case of metastatic urethral adenocarcinoma that showed a significant response to nabpaclitaxel.

\section{Case Report}

A 67-year-old Hispanic woman diagnosed with advanced metastatic adenocarcinoma of the urethra presented to our institute for a second opinion. Her condition started with symptoms of frequency, urgency, urinary incontinence, and pelvic discomfort. She was initially treated for urge incontinence but subsequently developed acute urine retention prompting further investigation. Physical examination revealed a firm submucosal periurethral mass measuring $3 \mathrm{~cm}$ in diameter inseparable from the anterior vaginal wall. Abdominal and pelvic CT scan and MRI showed a circumferential periurethral mass with evidence of metastasis to the pelvis, peritoneum, liver, bone, and pelvic lymph nodes. Cystoscopy failed to show any intraluminal pathology. CTguided biopsy revealed moderately differentiated adenocarcinoma of the urethra. She was started on 5 cycles of ifosfamide, paclitaxel, and cisplatin (ITP regimen). CT scan and MRI preformed after therapy showed mild decrease in the size of the metastases suggestive of partial response to therapy. Nevertheless, after 6 months her disease progressed and she was referred to hospice care, which the patient declined.

She presented to our institute for a second opinion. Tissue biopsy was obtained and sent for molecular profiling to help direct therapy. Molecular profiling displayed overexpression of SPARC, TOPO1, c-kit, and PDGFRB and under expression 


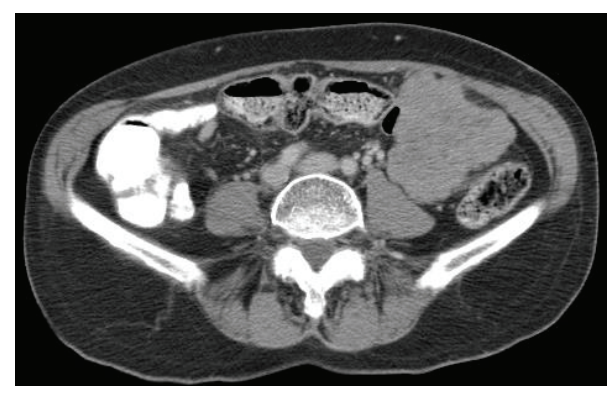

(a)

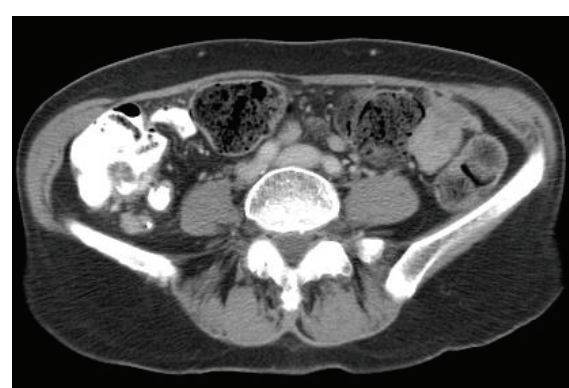

(b)

FIGURE 1: The figure shows the dramatic response of metastatic urethral cancer to nanoparticle albumin-bound (nab) paclitaxel. CT scan performed before therapy (a) showed an $11.5 \mathrm{~cm}$ metastatic mass in the left pelvis. This mass decreased to $3.5 \mathrm{~cm}$ after 5 months of nabpaclitaxel (b).

of TS, ERCC1, and GART. Nab-paclitaxel was thought to be a good therapeutic option given the presence of SPARC overexpression which increases the concentration of paclitaxel in the tumor interstitium leading to selective tumor cell apoptosis.

However, due to insurance issues, the patient was initiated on modified IFL chemotherapy which consists of irinotecan $\left(125 \mathrm{mg} / \mathrm{m}^{2}\right), 5$-fluorouracil $\left(500 \mathrm{mg} / \mathrm{m}^{2}\right)$, and leucovorin $\left(20 \mathrm{mg} / \mathrm{m}^{2}\right)$. After 8 cycles, her disease progressed and weekly nab-paclitaxel $\left(100 \mathrm{mg} / \mathrm{m}^{2}\right)$ was initiated. This dose was based on the results of two studies conducted on patients with metastatic breast cancer. A phase II study performed by Blum et al. compared $100 \mathrm{mg} / \mathrm{m}^{2}$ to $125 \mathrm{mg} / \mathrm{m}^{2}$ weekly nabpaclitaxel in patients with metastatic breast cancer that have been heavily pretreated with taxanes. This study showed that weekly administration of $100 \mathrm{mg} / \mathrm{m}^{2}$ nab-paclitaxel was as effective as $125 \mathrm{mg} / \mathrm{m}^{2}$ and had a more favorable side effect profile [7]. Another randomized phase III study conducted by Seidman et al. found that weekly administration of paclitaxel was more effective than triweekly in patients with metastatic breast cancer [8]. Given that our patient was heavily pretreated with taxanes we found this regimen to be the most suitable for her condition.

Nab-paclitaxel was well tolerated except for the development of grade 1 peripheral neuropathy and chemotherapyinduced anemia. Dramatic improvement was noted after 5 months of therapy with $70 \%$ reduction in the size of the pelvic masses on CT scan (Figure 1). Nab-paclitaxel was continued until disease progression providing about 19 months of progression free survival. Ultimately, nab-paclitaxel was discontinued and pemetrexed $\left(500 \mathrm{mg} / \mathrm{m}^{2}\right)$ introduced.

\section{Discussion}

Primary urethral cancer is three times more common in males than females, with a reported incidence rate of 4.3 per million and 1.5 per million, respectively [2]. Diagnosing urethral cancer can be a challenge requiring thorough physical examination under anesthesia, cystourethroscopy, and either CT scan or MRI of the abdomen and pelvis [3]. Biopsy of suspicious lesions is essential for establishing the diagnosis and determining the histological subtype. Prognosis is poor and depends largely upon the stage and location of the tumor. Unlike tumors of the proximal urethral, tumors of the distal urethra have better outcomes given their greater accessibility for resection and earlier presentation [3].

Literature available on primary urethral cancer is scarce and based mainly on small case series and reports. Given its rarity, histological heterogeneity, and advanced stage at presentation, no consensus exists regarding the optimal systemic therapy. The chemotherapeutic regimen used is based mainly upon the underlying histological subtype. Cisplatin and 5-fluorouracil are the most commonly used regimens for SCC $[3,9]$. TCC is treated like metastatic urothelial cancer using gemcitabine and cisplatin [10]. However, there is controversy regarding the optimal regimen for advanced urethral adenocarcinoma. A retrospective study conducted by Dayyani et al. reported efficacy of cisplatinbased chemotherapeutic regimens for patients with advanced urethral cancer, including SCC and adenocarcinoma [11]. The lack of optimal systemic chemotherapy for advanced urethral adenocarcinoma warrants the need to explore different novel agents in an attempt to improve outcomes.

Nab-paclitaxel, a Cremophor-free albumin-bound $130 \mathrm{~nm}$ particle form of paclitaxel, was approved by the Food and Drug Administration in 2005 for the treatment of metastatic breast cancer. Numerous studies have showed its efficacy in the treatment of metastatic breast, pancreatic, and bladder cancer [4-6]. The effectiveness of nab-paclitaxel is thought to be due to SPARC-albumin interaction. SPARC, a $43 \mathrm{kDa}$ secreted protein, is a key regulator for numerous cellular functions including cell proliferation, survival, and cell migration. It is secreted by both cancer and stromal cells and is therefore highly expressed in the tumor-stromal interface of invading tumors. It has a high affinity for albumin and is induced by both hypoxia and acidity. Many tumors accumulate albumin for de novo protein synthesis. Being albumin-bound, nab-paclitaxel uses the gp60 and caveolae-mediated albumin transport pathway to traverse the endothelial lining of blood vessels entering the tumor interstitium where it is trapped by SPARC. As a result, the concentration of paclitaxel near tumor cells increases thereby inducing their selective apoptosis. Although SPARC 
overexpression is associated with tumor progression and poor prognosis, targeted therapy using nab-paclitaxel may improve outcomes in these patients [12].

In our patient, molecular profiling showed SPARC overexpression and, hence, the dramatic response to nabpaclitaxel. This underscores the significance of molecular studies in predicting prognosis and response to treatment, thereby guiding the selection of chemotherapeutic regimens. This is the first case report to document the use of nabpaclitaxel in the treatment of unresectable metastatic urethral cancer. Further studies are needed to assess the efficacy of nab-paclitaxel as first or second-line treatment for patients with advanced urethral cancer.

\section{Abbreviations}

TCC: Transitional cell carcinoma

SCC: $\quad$ Squamous cell carcinoma

Nab-paclitaxel: Nanoparticle albumin-bound-paclitaxel

SPARC: $\quad$ Secreted protein, acidic, cysteine-rich

TOPO1: $\quad$ Topoisomerase inhibitor 1

PDGFRB: $\quad$ Platelet-derived growth factor receptor, beta polypeptide

TS: $\quad$ Thymidylate synthase

ERCC1: Excision repair cross-complementation group 1

GART: $\quad$ Glycinamide ribonucleotide transformylase

CT: $\quad$ Computed tomography

MRI: $\quad$ Magnetic resonance imaging.

\section{Conflict of Interests}

The authors declare that there is no conflict of interests regarding the publication of this paper.

\section{References}

[1] X. Wang, P. Bai, H. Su, G. Luo, Z. Zhong, and X. Zhao, "Management of primary adenocarcinoma of the female urethra: report of two cases and review of the literature," Oncology Letters, vol. 4, no. 5, pp. 951-954, 2012.

[2] M. A. Swartz, M. P. Porter, D. W. Lin, and N. S. Weiss, "Incidence of primary urethral carcinoma in the United States," Urology, vol. 68, no. 6, pp. 1164-1168, 2006.

[3] P. D. Grivas, M. Davenport, J. E. Montie, L. P. Kunju, F. Feng, and A. Z. Weizer, "Urethral Cancer," Hematology/Oncology Clinics of North America, vol. 26, no. 6, pp. 1291-1314, 2012.

[4] W. J. Gradishar, D. Krasnojon, S. Cheporov et al., "Phase II trial of nab-paclitaxel compared with docetaxel as first-line chemotherapy in patients with metastatic breast cancer: final analysis of overall survival," Clinical Breast Cancer, vol. 12, no. 5, pp. 313-321, 2012.

[5] D. D. Von Hoff, T. Ervin, F. P. Arena et al., "Increased survival in pancreatic cancer with nab-paclitaxel plus gemcitabine," The New England Journal of Medicine, vol. 369, no. 18, pp. 1691-1703, 2013.

[6] Y.-J. Ko, C. M. Canil, S. D. Mukherjee et al., "Nanoparticle albumin-bound paclitaxel for second-line treatment of metastatic urothelial carcinoma: a single group, multicentre, phase 2 study," The Lancet Oncology, vol. 14, no. 8, pp. 769-776, 2013.

[7] J. L. Blum, M. A. Savin, G. Edelman et al., "Phase II study of weekly albumin-bound paclitaxel for patients with metastatic breast cancer heavily pretreated with taxanes," Clinical Breast Cancer, vol. 7, no. 11, pp. 850-856, 2007.

[8] A. D. Seidman, D. Berry, C. Cirrincione et al., "Randomized phase III trial of weekly compared with every-3-weeks paclitaxel for metastatic breast cancer, with trastuzumab for all HER2 overexpressors and random assignment to trastuzumab or not in HER-2 nonoverexpressors: final results of cancer and leukemia group B protocol 9840," Journal of Clinical Oncology, vol. 26, no. 10, pp. 1642-1649, 2008.

[9] J. Itoh, K. Mitsuzuka, S. Kimura et al., "cisplatin and 5fluorouracil chemotherapy with concurrent radiation for unresectable advanced urethral carcinoma," International Journal of Urology, vol. 21, no. 4, pp. 422-424, 2014.

[10] H. von der Maase, L. Sengelov, J. T. Roberts et al., "Long-term survival results of a randomized trial comparing gemcitabine plus cisplatin, with methotrexate, vinblastine, doxorubicin, plus cisplatin in patients with bladder cancer," Journal of Clinical Oncology, vol. 23, no. 21, pp. 4602-4608, 2005.

[11] F. Dayyani, C. A. Pettaway, A. M. Kamat, M. F. Munsell, K. Sircar, and L. C. Pagliaro, "Retrospective analysis of survival outcomes and the role of cisplatin-based chemotherapy in patients with urethral carcinomas referred to medical oncologists," Urologic Oncology, vol. 31, no. 7, pp. 1171-1177, 2013.

[12] N. Desai, V. Trieu, B. Damascelli, and P. Soon-Shiong, "SPARC expression correlates with tumor response to albumin-bound paclitaxel in head and neck cancer patients," Translational Oncology, vol. 2, no. 2, pp. 59-64, 2009. 


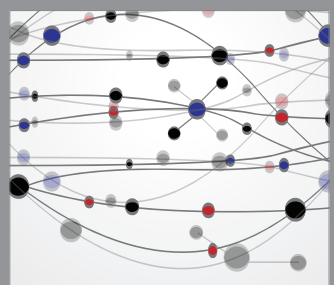

The Scientific World Journal
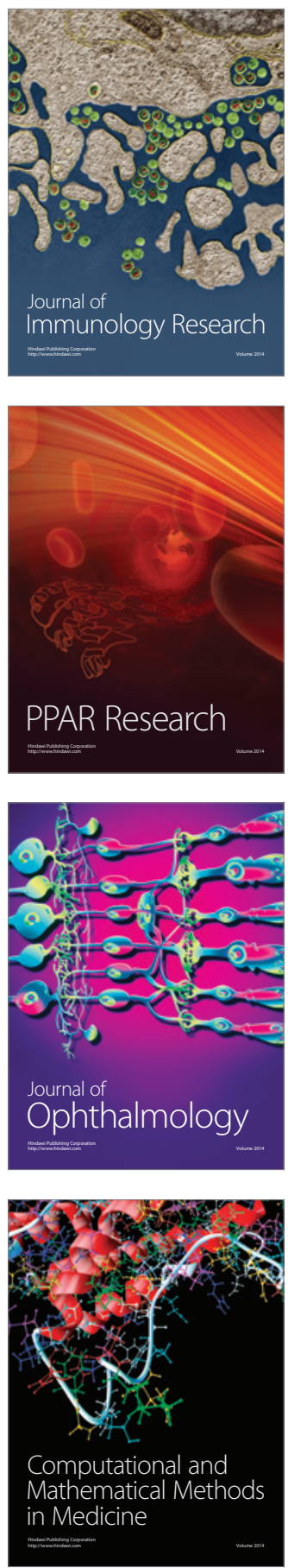

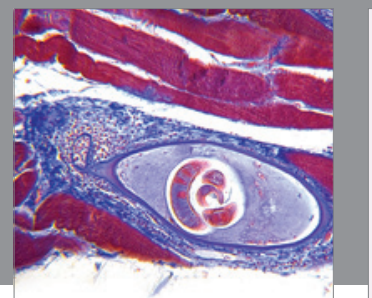

Gastroenterology

Research and Practice
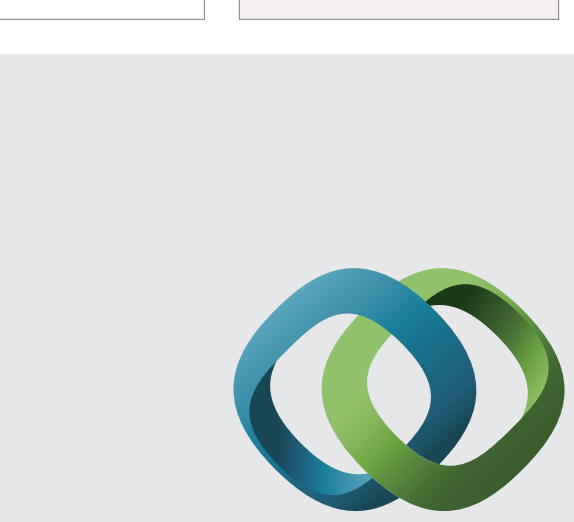

\section{Hindawi}

Submit your manuscripts at

http://www.hindawi.com
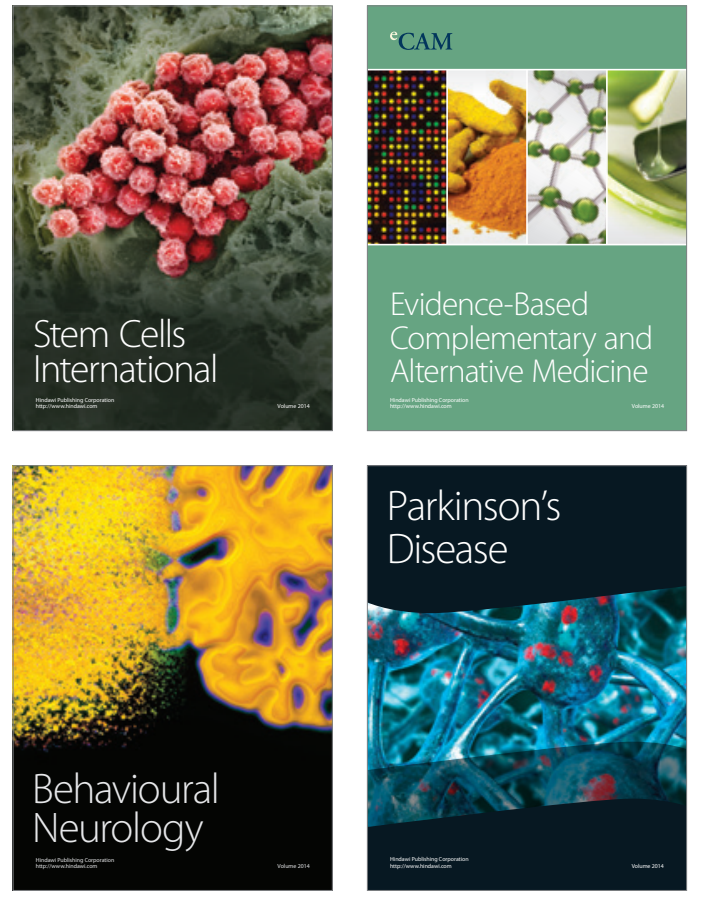
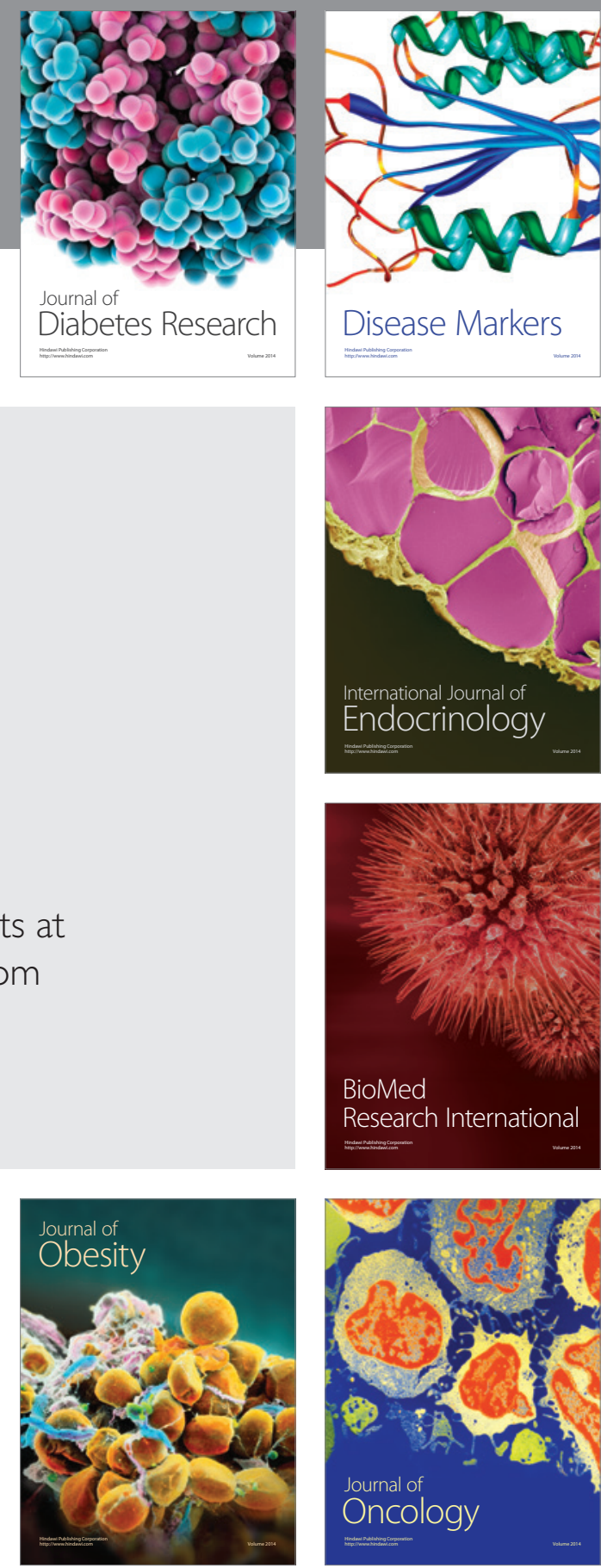

Disease Markers
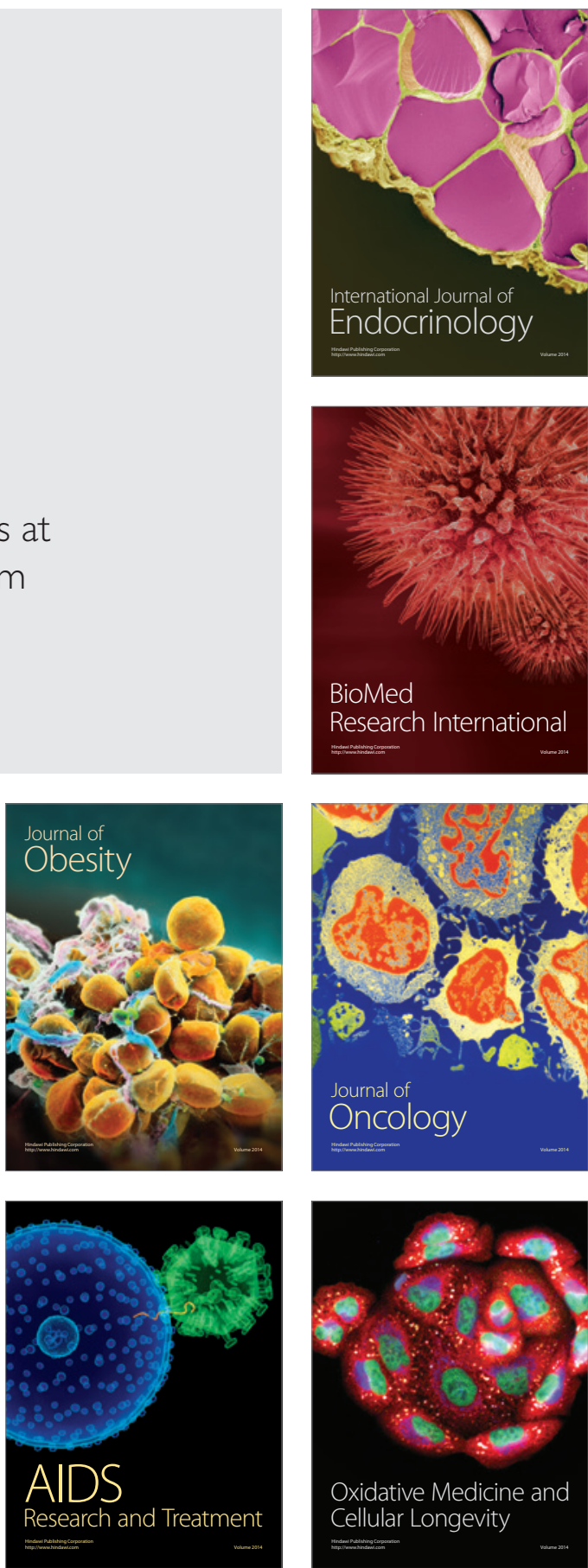